\title{
L'émigration des médecins titulaires d'un diplôme fédéral
}

\section{Maria Hodel, Daniel Kauer}

Unité de direction Politique de la santé, Office fédéral de la santé publique OFSP

\section{Correspondance:}

Maria Hodel

Office fédéral de la santé publique (OFSP)

Schwarzenburgstrasse 161

CH-3003 Berne

Tél. 0313238787

Fax 0313238805

maria.hodel[at]bag.admin.ch

\section{Introduction}

Le présent article résume les résultats d'un sondage qui traite un aspect rarement étudié du mouvement migratoire international, à savoir l'émigration de médecins formés en Suisse. Jusqu'à présent, cette discussion est fortement marquée par le fait que la Suisse accueille un grand nombre de médecins formés à l'étranger. A l'inverse, il n'y a guère d'informations, ni de collecte systématique de données, en ce qui concerne les médecins, formés en Suisse, qui quittent notre système de soins de santé. Ce sujet n'est pourtant pas dénué d'importance si l'on veut mener un débat politique pondéré sur la migration du personnel de santé ou justifier des décisions politiques telles que l'évaluation des besoins futurs de formation en médecine humaine.

C'est pour cette raison que l'Office fédéral de la santé publique (OFSP) a décidé d'effectuer un sondage visant à répondre aux questions suivantes: Combien de médecins formés en Suisse décident-ils de quitter le système suisse de santé, à titre temporaire ou définitif? Quels sont leurs pays de destination? Quels buts cherchent-ils à atteindre à l'étranger?

\section{Procédé}

En règle générale, les médecins qui souhaitent exercer ou être admis dans un institut de formation à l'étranger doivent présenter un certificat de conformité aux directives de l'UE ou une traduction de leur diplôme, délivrée par l'OFSP (document administratif) [1]. Pour en savoir davantage sur leurs projets à l'étranger, il a été décidé d'adresser pendant un an, à tous les médecins demandant un tel document auprès de l'OFSP, un questionnaire standard relatif à leur projet d'émigration.

Entre mai 2011 et mai 2012, ils ont été 222 médecins à solliciter un document administratif (échantillon). Parmi eux, 140 personnes ont complété le questionnaire, soit un taux de réponse de 63,1\%. De 2007

\section{Tableau 1}

Données chiffrées de l'échantillon (réponses reçues).

\begin{tabular}{lcll} 
& Hommes & Femmes & Total \\
\hline Questionnaires envoyés (réponses reçues) & $130(84)$ & $92(56)$ & $222(140)$ \\
\hline Avec au moins un titre de spécialisation & $80(58)$ & $40(27)$ & $120(85)$ \\
\hline Durchschnittsalter (réponses reçues) & $38,4(39)$ & $34,7(35.5)$ & $36,9(37,6)$
\end{tabular}

à 2009, l'OFSP a établi quelque 200 à 250 documents administratifs chaque année, aussi la taille de l'échantillon étudié s'avère-t-elle représentative [2]. Le tableau 1 ci-dessous détaille, par sexe, la distribution du nombre des répondants titulaires d'un diplôme de spécialisation ainsi que leur âge moyen.

\section{Résultats}

Séjours à l'étranger envisagés et durée prévue Le tableau 2 fait apparaître que 123 des 140 répondants ont l'intention d'émigrer à titre temporaire ou pour une durée indéterminée. 17 personnes déclarent avoir besoin de la confirmation pour raisons administratives à l'intérieur du pays (par ex., pour s'inscrire à un examen de spécialiste). Pour 22 personnes, le séjour à l'étranger durera moins de six mois et s'explique par un engagement humanitaire, la participation à des manifestations sportives ou l'acceptation d'une charge de cours. Certaines d'entre elles comptent d'ailleurs travailler tant en Suisse qu'à l'étranger (par ex., comme frontalier). Compte tenu de la durée réduite du séjour à l'étranger ou de l'hétérogénéité des activités prévues, ce groupe a été exclu de l'analyse ultérieure. Il en va de même pour deux personnes qui n'ont pas mentionné d'indication de durée pour leur séjour à l'étranger.

\section{Activités prévues à l'étranger selon le degré de formation}

Pour l'étude des activités prévues à l'étranger, nous n'avons pris en considération que les personnes qui annonçaient un séjour à l'étranger d'une durée supérieure à 6 mois. Il s'agit de 99 personnes, dont 54 disposent déjà d'un titre postgrade. Pour décrire les activités prévues, les répondants avaient le choix entre plusieurs types de réponse, classés en quatre catégories:activités de formation exclusivement (B); exercice de la profession exclusivement (BA); activités de recherche exclusivement $(\mathrm{F})$; combinaison d'activités de formation, d'exercice de la profession et de recherche $(\mathrm{B} / \mathrm{B} / \mathrm{F})$.

Le graphique ci-dessous (fig.1) présente les données classées conformément à ces catégories, en distinguant les médecins selon qu'ils sont ou non titulaires d'un titre postgrade.

Comme on pouvait s'y attendre, l'analyse montre que les médecins sans titre postgrade partent plus souvent à l'étranger pour poursuivre leur forma- 
Figure 1

Distribution des activités prévues selon le degré de formation $(N=99)$.

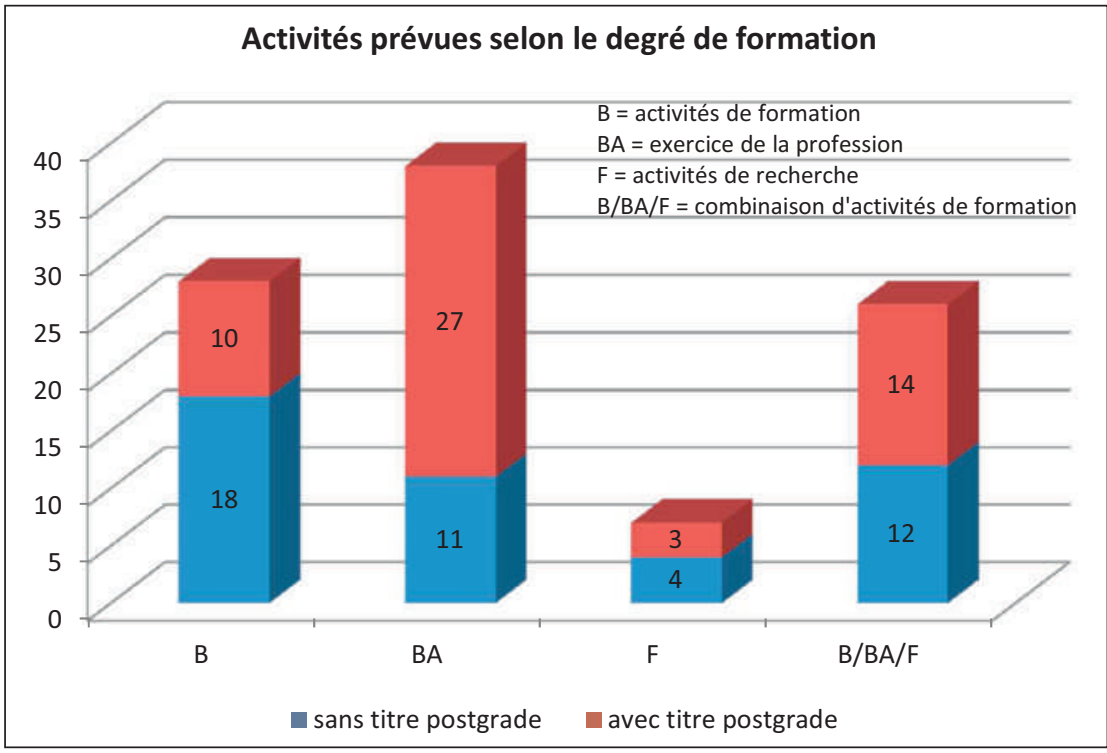

Tableau 2

Durée prévue du séjour à l'étranger $(\mathrm{N}=140)$.

\begin{tabular}{lccc} 
Durée du séjour à l'etranger & $\begin{array}{l}\text { Pas de séjour à } \\
\text { l'étranger }(\mathrm{N})\end{array}$ & $\begin{array}{l}\text { Séjour à } \\
\text { l'étranger }(\mathrm{N})\end{array}$ & en \% \\
\hline 0 mois & 17 & 22 & 12,1 \\
\hline jusqu'à 6 mois & & 26 & 15,7 \\
\hline de 6 mois à 1 an & 52 & 18,6 \\
\hline plusieurs années & & 21 & 37,2 \\
\hline durée indéterminée & 17 & 123 & 15 \\
\hline non divulgué & & & 100 \\
\hline Total & & &
\end{tabular}

Figure 2

Principaux pays de destination et activités prévues $(\mathrm{N}=79)$.

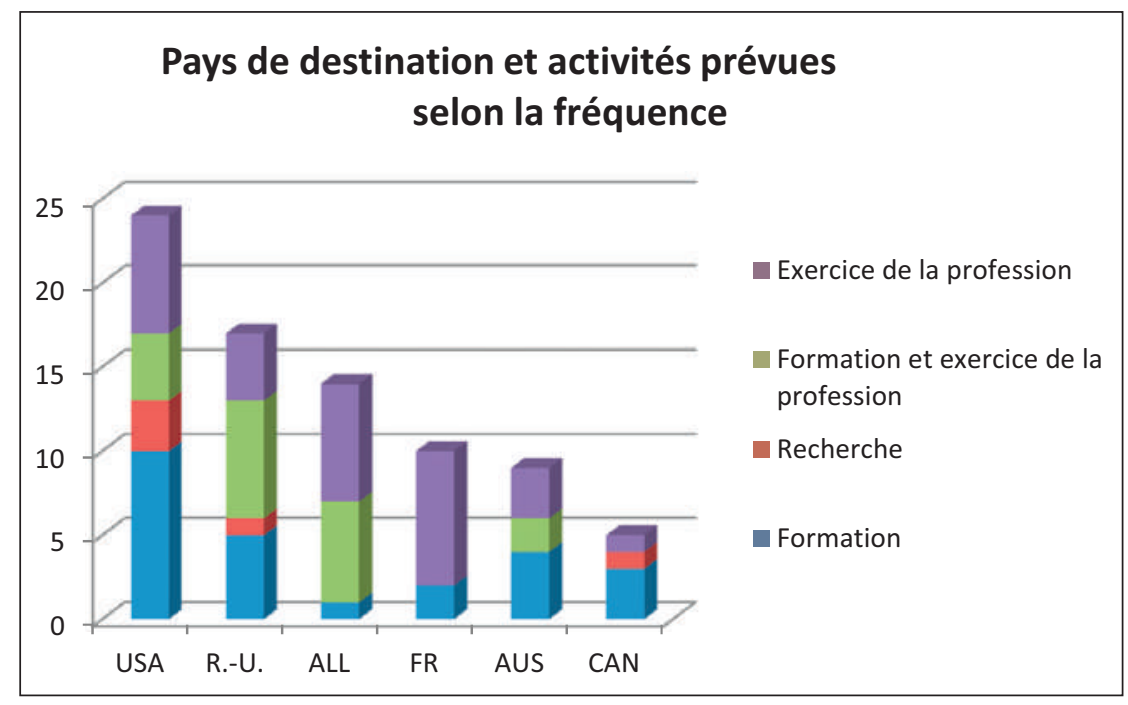

tion. C'est encore plus frappant lorsque l'on considère le groupe de ceux qui déclarent partir à l'étranger exclusivement dans le but d'y exercer leur profession. Quant au nombre de médecins déclarant partir à l'étranger exclusivement pour se livrer à la recherche, il est relativement réduit. Près d'un quart des répondants prévoit de se livrer à une combinaison de ces activités.

Pays de destination classés par fréquence et par type d'activité prévue

Les 123 répondants désireux de quitter la Suisse mentionnent, au total, 23 pays de destination distincts. Le graphique ci-dessous (graphique 2) présente une analyse pour les six pays principaux de destination et les activités qui y sont prévues.

En matière de formation ou de recherche, les pays de destination les plus souvent cités sont les pays anglo-saxons. Les motivations les plus fréquemment évoquées pour justifier le départ à l'étranger sont l'accès à un nombre plus élevé de cas, ou encore les opportunités de formation et de carrière ainsi que la culture professionnelle généralement plus favorables.

\section{Synthèse et discussion}

Chaque année, de 200 à 250 médecins introduisent auprès de l'OFSP une demande de documents administratifs généralement nécessaires pour exercer une activité à l'étranger. Ces médecins ont fait l'objet d'une étude succincte, plutôt exploratoire, sur leur projet d'émigration, en vue d'approfondir la discussion politique sur les besoins de formation en médecine humaine. Les résultats obtenus montrent que près de $88 \%$ des répondants (123 sur 140) envisagent effectivement un séjour à l'étranger. Si l'on rapporte ces résultats à l'intégralité de l'échantillon [3] ( $\mathrm{N}=$ 222), cela signifie que près de 200 médecins sont susceptibles de quitter la Suisse chaque année. Quelque 120 d'entre eux envisagent un séjour à l'étranger de plusieurs années, voire de durée indéterminée. Les 80 autres répondants ne prévoient pas de séjourner à l'étranger plus d'un an. Les médecins formés en Suisse privilégient les pays anglo-saxons; l'Allemagne et la France, pays limitrophes, sont également des destinations prisées. Les mouvements migratoires des médecins formés en Suisse s'inscrivent ainsi dans le flux migratoire mondial des professionnels de la santé, du Sud au Nord et de l'Est vers l'Ouest.

Le nombre de candidats à l'émigration est faible compte tenu du nombre de diplômes de médecine en provenance de l'espace UE/EEE reconnus par l'OFSP, à savoir 1930 diplômes de médecine en 2011, et même 1769 à fin septembre 2012. Par comparaison, 200 médecins représentent environ un quart de l'effectif moyen du nombre de médecins formés chaque année en Suisse. C'est une proportion élevée et donc impressionnante. Elle démontre la grande 
propension à la mobilité des médecins formés ici, ainsi que la grande importance des échanges internationaux, en particulier avec des pays anglosaxons.

Les résultats de la présente étude prennent toute leur importance dans le cadre du débat en cours sur les besoins de formation et sur les capacités optimales d'accueil à mettre en place dans les universités cune donnée concrète relative à la fréquence, aux motivations et à la durée des séjours à l'étranger n'existait encore. Les résultats de la présente étude confortent encore la recommandation du Conseil fédéral visant à augmenter le nombre de diplômes en médecine humaine.

\section{Le nombre de candidats à l'émigration est faible compte tenu du nombre de diplômes de médecine en provenance de l'espace UE/EEE.}

suisses. Dans son rapport «Stratégie pour lutter contre la pénurie de médecins et encourager la médecine de premier recours», le Conseil fédéral recommandait d'augmenter d'environ 800 à 1200 ou 1300 le nombre de diplômes décernés chaque année: crest le nombre de médecins qui est indispensable pour maintenir les capacités actuellement disponibles. Ce calcul tient compte des déperditions probables observées au terme de la formation [4] de même que la réduction présumée des prestations médicales disponibles suite à la féminisation de la profession (diminution du taux d'occupation). Dans le rapport du Conseil fédéral, la déperdition d'années de travail productif encourue par la Suisse à cause de l'émigration n'est qu'évoquée, elle n'est pas prise en compte. Au moment de sa publication, au-

\section{Références}

1 Les certificats de conformité aux directives sont indispensables pour être autorisé à poursuivre une formation postgrade ou à exercer la profession dans des pays de l'UE/de l'EEE. La traduction du diplôme en langue anglaise est essentiellement demandée pour des séjours aux Etats-Unis ou au Canada.

2 Un graphique analogue a été publié dans le rapport du Conseil fédéral intitulé «Stratégie pour lutter contre la pénurie de médecins et encourager la médecine de premier recours», page 31. Voir: www.bag.admin.ch/ themen/berufe/07962/index.html?lang=fr

4 L'échantillon représente le nombre de médecins qui, au cours d'une année, ont demandé à l'OFSP soit une attestation de conformité aux directives, soit une traduction en langue anglaise de leur diplôme.

5 Il s'agit de médecins qui n'ont jamais suivi de formation postgrade. 